\title{
Author Correction: Nested designs
}

Martin Krzywinski, Naomi Altman and Paul Blainey

Correction to: Nature Methods https://doi.org/10.1038/nmeth.3137, published online 29 September 2014.

In the version of this article initially published, there were errors in an $n$ value, two subscripts and a figure citation. In the sentence in paragraph 11 beginning "If variance is kept at $1.43(c=5, n=5)$," $n$ should be 3 , not 5 . In the sentence in paragraph 12 beginning "We want to minimize $\operatorname{Var}\left(X_{i} \ldots\right)=\sigma_{\mathrm{B}}^{2} / b+\sigma_{\mathrm{C}}^{2} /(b c)+\sigma_{\varepsilon}^{2} /(b c n)$ within the cost constraint $K=b C_{\mathrm{B}}+b c C_{\mathrm{D}}+b c n C_{\mathrm{D}}$," the subscript $\mathrm{D}$ in the second term should be a subscript $\mathrm{C}$ and the one in the third should be a subscript $\mathrm{N}$. In the next-to-last paragraph, the citation to Fig. $2 \mathrm{~b}$ should be to Fig. 3b. The authors thank Ghadi Al Hajj for bringing the matter to their attention. The errors have not been corrected in the original article.

Published online: 9 September 2020

https://doi.org/10.1038/s41592-020-0969-7

() Springer Nature America, Inc. 2020

\section{Publisher Correction: High-sensitivity and high-specificity biomechanical imaging by stimulated Brillouin scattering microscopy}

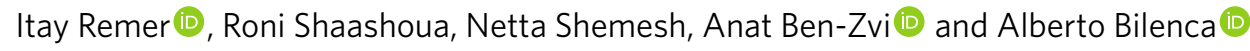

Correction to: Nature Methods https://doi.org/10.1038/s41592-020-0882-0, published online 3 August 2020.

In the version of this article initially published online, the gray wavefronts representing acoustic resonance were missing from the right panel of Fig. 1a, and an extra letter A was present to the left of the L2 larva in Fig. 2h. The errors have been corrected in the print, PDF and HTML versions of the article.

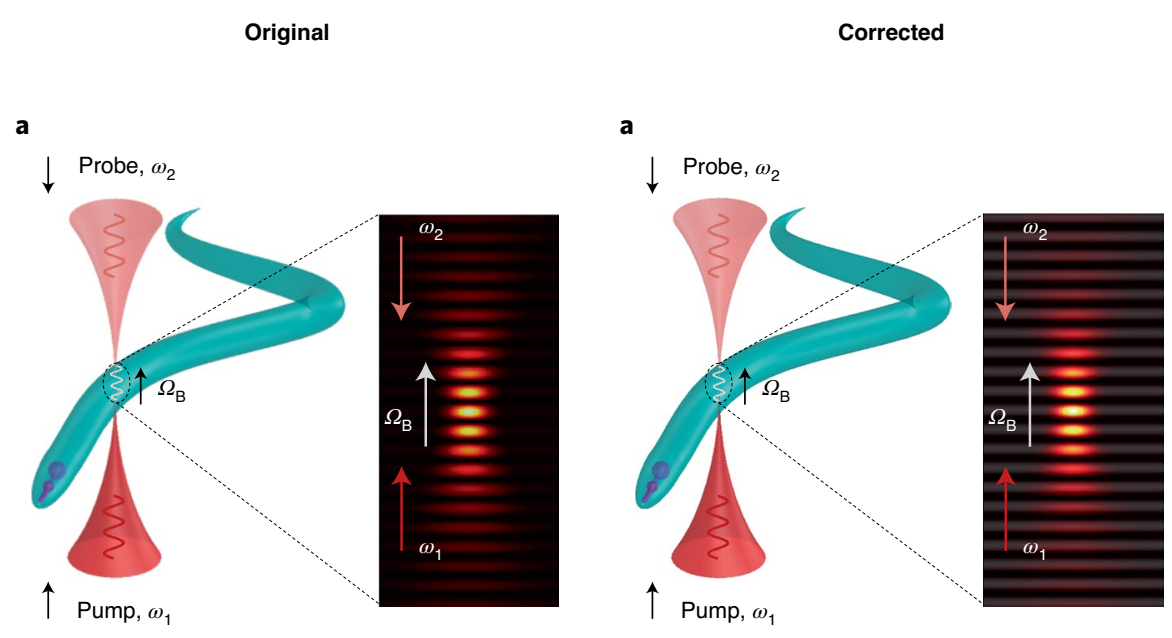

Fig. 1 | Original and corrected.

Published online: 20 August 2020

https://doi.org/10.1038/s41592-020-0956-Z

( $)$ The Author(s), under exclusive licence to Springer Nature America, Inc. 2020 